\title{
Dynamical chiral symmetry breaking by a magnetic field and multi-quark interactions
}

\author{
A.A. Osipov ${ }^{\mathrm{a}, *}$, B. Hiller ${ }^{\mathrm{b}}$, A.H. Blin ${ }^{\mathrm{b}}$, J. da Providência ${ }^{\mathrm{b}}$ \\ a Dzhelepov Laboratory of Nuclear Problems, JINR, 141980 Dubna, Russia \\ ${ }^{\mathrm{b}}$ Centro de Física Teórica, Departamento de Física da Universidade de Coimbra, 3004-516 Coimbra, Portugal
}

Received 11 January 2007; received in revised form 14 March 2007; accepted 20 May 2007

Available online 24 May 2007

Editor: L. Alvarez-Gaumé

\begin{abstract}
Catalysis of dynamical symmetry breaking by a constant magnetic field in $(3+1)$ dimensions is considered. We use the three flavour NambuJona-Lasinio type model with 't Hooft and eight-quark interaction terms. It is shown that the multi-quark interactions introduce new additional features to this phenomenon: (a) the local minimum of the effective potential catalyzed by the constant magnetic field is smoothed out with increasing strength of the field at the characteristic scale $H \sim 10^{19} \mathrm{G}$, (b) the multi-quark forces generate independently another local minimum associated with a larger dynamical fermion mass. This state may exist even for multi-quark interactions with a subcritical set of couplings, and is globally stable with respect to a further increase of the magnetic field.
\end{abstract}

(c) 2007 Elsevier B.V. All rights reserved.

PACS: 11.30.Rd; 11.30.Qc

It has been shown in a series of papers [1-3] that in $(2+1)$ and $(3+1)$ dimensions a constant magnetic field $H \neq 0$ catalyzes the dynamical symmetry breaking leading to a fermion mass even at the weakest attractive four-fermion interaction between particles, and the symmetry is not restored at any arbitrarily large $H$. Soon thereafter it became also clear [4-6] that the zero-energy surface of the lowest Landau level (LLL) plays a crucial role in the dynamics of such fermion pairing. It has been found that the dynamics of the fermion pairing in the homogeneous magnetic field is essentially $(1+1)$-dimensional, and a deep analogy of this phenomenon with the dynamics of electron pairing in BCS [7] has been stressed. The generated fermion mass, $M_{\text {dyn }}$, turned out to be much smaller than the Landau gap $\sim \sqrt{|e H|}$.

The existence of a zero-energy surface in the spectrum of a Dirac particle is ensured for any homogeneous magnetic field with a fixed direction by a quantum mechanical supersymme-

\footnotetext{
* Corresponding author.

E-mail address: osipov@nu.jinr.ru (A.A. Osipov).
}

try of the corresponding second-order Dirac Hamiltonian [8]. This aspect of the phenomenon appears to be a quite exceptional situation and indicates that the dynamical generation of mass is not so universal as one would expect by extrapolating the results obtained for homogeneous or unidirectional [9] magnetic field profiles. For instance, it has been demonstrated by Ragazzon [10] that the Nambu-Jona-Lasinio (NJL) model [11] minimally coupled to a background magnetic field with variable direction does not possess a massive phase until the coupling constant exceeds some critical value. Obviously, in this case one faces the conventional scenario of dynamical chiral symmetry breaking, where the magnetic field does not play an essential role.

Conversely, having in mind that homogeneous magnetic fields can act as strong catalysts of chiral symmetry breaking, one might ask what is the effect caused by the strong interaction, when higher order multi-fermion interactions are present. These extensions of the NJL model are well known [12-14], for instance, the four-quark $U(3)_{L} \times U(3)_{R}$ chiral symmetric Lagrangian together with the $U(1)_{A}$ breaking 't Hooft six-quark interactions has been extensively studied at the mean-field level 
[15-18]. Recently it has been also shown $[19,20]$ that the eightquark interactions are of vital importance to stabilize the multiquark vacuum.

The additional multi-quark forces can affect the result which is obtained when only four-fermion interactions are considered. We argue, in particular, that the 't Hooft and eight-quark interactions can modify the theory in such a way that the local minimum, catalyzed by the constant magnetic field, is smoothed out by increasing the strength of the field. This is an alternative regime to the known one in which the strong magnetic field cannot wash out the condensate from the LLL. For the first scenario to become possible it is sufficient that the couplings of multi-quark interactions are chosen such that the system displays more than one solution of the gap equation at $H=0$. Actually, the above condition is not a requirement. Even if the gap equation has only one nontrivial solution at small $H$, an increase in the magnetic field can induce the formation of a second minimum.

The multi-quark dynamics of the extended NJL model is described by the Lagrangian density

$\mathcal{L}_{\text {eff }}=\bar{q}\left(i \gamma^{\mu} D_{\mu}-\hat{m}\right) q+\mathcal{L}_{4 q}+\mathcal{L}_{6 q}+\mathcal{L}_{8 q}$,

where the gauge covariant derivative $D_{\mu}$ is equal to $D_{\mu}=$ $\partial_{\mu}+i Q A_{\mu}$ with the external electromagnetic field $A_{\mu}$ and quark charges $Q=e \cdot \operatorname{diag}(2 / 3,-1 / 3,-1 / 3)$. It is assumed that quark fields have colour $\left(N_{c}=3\right)$ and flavour $\left(N_{f}=3\right)$ indices. The current quark mass, $\hat{m}$, is a diagonal matrix with elements $\operatorname{diag}\left(\hat{m}_{u}, \hat{m}_{d}, \hat{m}_{s}\right)$, which explicitly breaks the global chiral $S U_{L}(3) \times S U_{R}(3)$ symmetry of the Lagrangian. We shall neglect this effect in the following assuming that $\hat{m}=0$.

The multi-quark interactions (in the scalar and pseudoscalar channels) are

$$
\begin{aligned}
& \mathcal{L}_{4 q}=\frac{G}{2}\left[\left(\bar{q} \lambda_{a} q\right)^{2}+\left(\bar{q} i \gamma_{5} \lambda_{a} q\right)^{2}\right], \\
& \mathcal{L}_{6 q}=\kappa\left(\operatorname{det} \bar{q} P_{L} q+\operatorname{det} \bar{q} P_{R} q\right), \\
& \mathcal{L}_{8 q}=\mathcal{L}_{8 q}^{(1)}+\mathcal{L}_{8 q}^{(2)} .
\end{aligned}
$$

The $U$ (3) flavour matrices $\lambda_{a}, a=0,1, \ldots, 8$, are normalized such that $\operatorname{tr}\left(\lambda_{a} \lambda_{b}\right)=2 \delta_{a b}$. The matrices $P_{L, R}=\left(1 \mp \gamma_{5}\right) / 2$ are chiral projectors and the determinant is over flavour indices, which are suppressed here. The determinantal interaction breaks explicitly the axial $U(1)_{A}$ symmetry [21] and Zweig's rule. The eight-quark spin zero interactions are given by

$\mathcal{L}_{8 q}^{(1)}=8 g_{1}\left[\left(\bar{q}_{i} P_{R} q_{m}\right)\left(\bar{q}_{m} P_{L} q_{i}\right)\right]^{2}$,

$\mathcal{L}_{8 q}^{(2)}=16 g_{2}\left(\bar{q}_{i} P_{R} q_{m}\right)\left(\bar{q}_{m} P_{L} q_{j}\right)\left(\bar{q}_{j} P_{R} q_{k}\right)\left(\bar{q}_{k} P_{L} q_{i}\right)$.

$G, \kappa, g_{1}, g_{2}$ are dimensionful coupling constants: $[G]=M^{-2}$, $[\kappa]=M^{-5},\left[g_{1}\right]=\left[g_{2}\right]=M^{-8}$ in units $\hbar=c=1$.

We proceed by calculating the effective potential of the theory, $V\left(m_{u}, m_{d}, m_{s}\right)$, in a constant magnetic field: $A_{x}=$ $-H y, A_{y}=A_{z}=0$ (Landau gauge). The arguments, $m_{i}$, are simply real parameters; they are not to be identified with the masses of any presumed one-particle states. Instead, we shall use the capital letter $M_{i}$ for the point where $V$ takes its local minimum, which specifies the masses of constituent quark fields.
The potential is built of the following two terms

$V\left(m_{u}, m_{d}, m_{s}\right)=V_{s t}+V_{S}$.

The first contribution results from the many-fermion vertices of Lagrangian $\mathcal{L}_{\text {eff }}$, after reducing them to a bilinear form with help of bosonic auxiliary fields, and subsequent integration over these fields, using the stationary phase approximation (SPA) method. The specific details of these calculations and the result are given in a recent work [19]. We obtain

$V_{s t}=\frac{1}{16}\left(4 G h^{2}+\kappa h_{u} h_{d} h_{s}+\frac{3 g_{1}}{2}\left(h^{2}\right)^{2}+3 g_{2} h^{4}\right)$,

where $h^{2}=\sum_{i=u, d, s} h_{i}^{2}$, and $h^{4}=\sum_{i=u, d, s} h_{i}^{4}$. The functions $h_{i}$ depend on the coupling constants $G, \kappa, g_{1}, g_{2}$ and on the independent variables $\Delta_{i}=m_{i}-\hat{m}_{i}$. To find this dependence one should solve the system of cubic equations

$G h_{i}+\Delta_{i}+\left.\frac{\kappa}{16} h_{j} h_{k}\right|_{j \neq k \neq i}+\frac{g_{1}}{4} h_{i} h^{2}+\frac{g_{2}}{2} h_{i}^{3}=0$.

In some parameter range the system has only one set of real solutions, and this guarantees the vacuum state of the theory to be stable [19].

The second term on the r.h.s. of Eq. (7) derives from the integration over the quark bilinears in the functional integral of the theory in presence of a constant magnetic field $H$. As has been calculated by Schwinger a long time ago [22]

$V_{S}=\sum_{i=u, d, s} V_{S}\left(m_{i},\left|Q_{i} H\right|\right)$,

where

$$
\begin{aligned}
& V_{S}(m,|Q H|) \\
& =\frac{N_{c}}{8 \pi^{2}} \int_{0}^{\infty} \frac{\mathrm{d} s}{s^{2}} e^{-s m^{2}} \rho\left(s, \Lambda^{2}\right)|Q H| \operatorname{coth}(s|Q H|)+\text { const. }
\end{aligned}
$$

Here the cutoff $\Lambda$ has been introduced by subtracting off suitable counterterms to regularize the integral at the lower limit, i.e., $\rho\left(s, \Lambda^{2}\right)=1-\left(1+s \Lambda^{2}\right) e^{-s \Lambda^{2}}$. For the fermion tadpole this works as the four-momentum covariant cutoff in the euclidean space: $\vec{p}^{2}+p_{4}^{2}<\Lambda^{2}$. The unessential constant is chosen such that $V_{S}(0,|Q H|)=0$. As a result we obtain

$$
\begin{aligned}
V_{S}( & m,|Q H|) \\
= & \frac{N_{c}}{8 \pi^{2}}\left\{\Lambda^{2}|Q H|\left[\ln 2 \pi-2 \ln \Gamma\left(\frac{\Lambda^{2}+m^{2}}{2|Q H|}\right)\right]\right. \\
& +m^{2}|Q H| \ln \left(1+\frac{\Lambda^{2}}{m^{2}}\right)+4(Q H)^{2} \\
& \times\left.\frac{\mathrm{d}}{\mathrm{d} v}\left[\zeta\left(\nu-1, \frac{\Lambda^{2}+m^{2}}{2|Q H|}\right)-\zeta\left(v-1, \frac{m^{2}}{2|Q H|}\right)\right]\right|_{\nu=0} \\
& \left.+\frac{\Lambda^{4}}{2}\left(\ln \frac{\Lambda^{2}}{2|Q H|}-\frac{3}{2}\right)-\Lambda^{2} m^{2}\right\} .
\end{aligned}
$$

The quantity $\zeta(v, x)$ denotes the generalized Riemann zeta function [23].

We shall now illustrate the procedure which will be employed in the following, by considering first the simple $S U(3)$ 


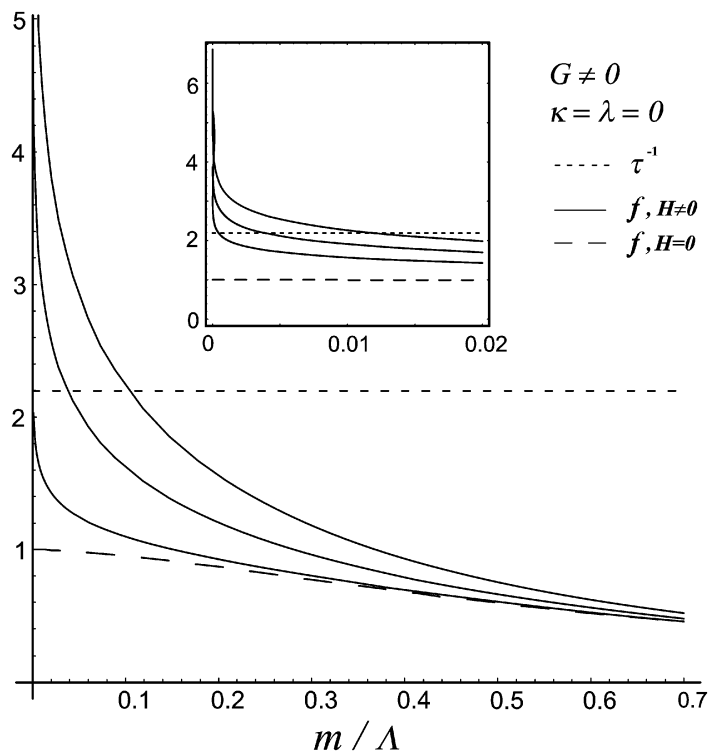

Fig. 1. The 1.h.s. (straight short-dashed line) and the r.h.s. of Eq. (13) as functions of $m / \Lambda$ for four different values of the magnetic field strength $H$ : full curves (top to bottom) correspond to $|Q H| \Lambda^{-2}=0.5 ; 0.3 ; 0.1$, and the dashed curve to $H=0$. Box insert: close-up of region around origin with solid lines for $|Q H| \Lambda^{-2}=0.2 ; 0.15 ; 0.1$ (top to bottom).

flavour limit for the situation in which $\hat{m}=0$ and $\kappa=g_{1}=$ $g_{2}=0$. For the purpose of illustration, we ignore the charge difference of $u$ and $d, s$ quarks in the remaining. The averaged common charge $|Q|=|4 e / 9|$ will be used. In this case one obtains the potential $V(m)=N_{f}\left(m^{2} / 4 G+V_{S}(m,|Q H|)\right)$. One sees that the gap equation, $\mathrm{d} V(m) / \mathrm{d} m=0$, has always a trivial solution, $m=0$. The nontrivial solution is contained in the equation

$$
\begin{aligned}
& \frac{2 \pi^{2}}{G \Lambda^{2} N_{c}} \\
& =f\left(m^{2} ; \Lambda,|Q H|\right) \\
& \equiv \psi\left(\frac{\Lambda^{2}+m^{2}}{2|Q H|}\right)-\frac{|Q H|}{\Lambda^{2}} \\
& \quad \times\left[\ln \left(1+\frac{\Lambda^{2}}{m^{2}}\right)-\frac{\Lambda^{2}}{\Lambda^{2}+m^{2}}+2 \ln \frac{\Gamma\left(\frac{\Lambda^{2}+m^{2}}{2|Q H|}\right)}{\Gamma\left(\frac{m^{2}}{2|Q H|}\right)}\right],
\end{aligned}
$$

where $\psi(x)=\mathrm{d} \ln \Gamma(x) / \mathrm{d} x$ is the Euler dilogarithmic function. This equation has a solution at all $G>0$, if $H \neq 0$. Fig. 1 illustrates this important result for $G \Lambda^{2}=3$. One sees that in absence of the magnetic field the system is in the subcritical regime of dynamical symmetry breaking. The introduction of a constant field, however small it might be, changes radically the dynamical symmetry breaking pattern, due to the singular behaviour of the r.h.s. of Eq. (13) close to the origin: the right- and left-hand sides will always intersect and the value of $m$ where this happens is a minimum of the effective potential.

Let us look at these different regimes more closely. As $H \rightarrow 0$, we recover the well-known NJL model gap equation

$$
1=\frac{G N_{c}}{2 \pi^{2}}\left(J_{0}\left(m^{2}\right)+\frac{|Q H|^{2} \Lambda^{2}}{3 m^{2}\left(\Lambda^{2}+m^{2}\right)}+\cdots\right),
$$

where $J_{0}\left(m^{2}\right)=\Lambda^{2}-m^{2} \ln \left(1+\Lambda^{2} / m^{2}\right)$. Eq. (14) at $H=0$ admits a nontrivial solution only if $\tau>1$, where $\tau=G \Lambda^{2} N_{c} / 2 \pi^{2}$. This determines the critical value $G_{\mathrm{cr}}=2 \pi^{2} / \Lambda^{2} N_{c}$.

At $m^{2} / \Lambda^{2} \ll 1$ the r.h.s. of Eq. (13) is

$-\frac{|Q H|}{\Lambda^{2}} \ln \left(\frac{m^{2}}{\Lambda^{2}}\right)+v(\xi)+\mathcal{O}\left(\frac{m^{2}}{\Lambda^{2}}\right)$.

Here the function $v(\xi)$ of the argument $\xi=\Lambda^{2} / 2|Q H|$, is given by

$v(\xi)=\frac{1}{2 \xi}[1-2 \ln \Gamma(\xi+1)]+\psi(\xi)$.

This is a monotonically increasing function on the interval $0<\xi<\infty ; v(\xi)=0$ at the point $\xi \simeq 1.12$; the asymptotic behaviour is

$v(\xi) \sim 1-\frac{1}{2 \xi} \ln (2 \pi \xi) \quad(\xi \rightarrow \infty)$,

$v(\xi) \sim-\gamma-\frac{1}{2 \xi} \quad(\xi \rightarrow 0)$,

where $\gamma \simeq 0.577$ is the Euler's constant.

In the approximation considered one finds the solution of Eq. (13)

$M_{\text {dyn }}=\Lambda \exp \left[-\xi\left(\frac{1}{\tau}-v(\xi)\right)\right]$.

To discuss the physical content of this result, we recall that the energy spectrum of relativistic fermions in a constant magnetic field $H$ contains Landau levels

$E_{n}\left(p_{z}\right)= \pm \sqrt{\hat{m}^{2}+2|Q H| n+p_{z}^{2}}, \quad n=0,1,2, \ldots$,

with $p_{z}$ denoting the projection of the 3-momentum on the $z$ axis, i.e., along the magnetic field. If the fermion mass $\hat{m}$ goes to zero, as in the present case, there is no energy gap between the vacuum and the LLL. Thus the integer part of $\xi+1$ gives approximately the number of Landau levels taken into account.

The first term in Eq. (15) has a clearly defined twodimensional origin, given the logarithmic dependence on the cutoff in the corresponding gap equation

$1=-\frac{G N_{c}}{2 \pi^{2}}|Q H| \ln \left(\frac{m^{2}}{\Lambda^{2}}\right)$

and, therefore, in the condensate (compare with Eq. (14)). Such behaviour is associated with the $(1+1)$-dimensional dynamics of the fermion pairing on the energy surface $E_{0}=0$ of the LLL [5]. As long as this term dominates over the second term, $v(\xi)$ in (15), one concludes that the condensate is mainly located on the LLL. Actually this condition is fulfilled nearly everywhere at $\tau<1$. Indeed, this is obvious for $\xi=1$, since $v(1)=1 / 2+\psi(1)=1 / 2-\gamma \simeq-0.08$ is small compared with $1 / \tau$. For $\xi<1$ we come to the same conclusion after considering the asymptotics of the second term (18). The other formula, (17), can be used to show that the above statement is also true for $\xi>1$, except near the critical region $\tau \rightarrow 1-0$, where $v(\xi)$ dominates; then the condensate spreads over many Landau levels. 
In this special case it is possible to find an analytical solution. Indeed, using (17) in Eq. (15) we obtain

$1-\frac{|Q H|}{\Lambda^{2}} \ln \left(\frac{\pi m^{2}}{|Q H|}\right)+\mathcal{O}\left(\frac{m^{2}}{\Lambda^{2}}, \frac{4|Q H|^{2}}{\Lambda^{4}}\right)$.

To progress further we suppose that the two following small variables are of the same order

$\frac{m^{2}}{\Lambda^{2}} \sim\left(\frac{|Q H|}{\Lambda^{2}}\right)^{2} \sim \epsilon$.

Then it follows immediately that the term with the logarithm is of order $\sqrt{\epsilon} \ln \sqrt{\epsilon}$ and goes to zero, when $\epsilon \rightarrow 0$. Thus, the gap equation

$1-\frac{1}{\tau}=\frac{|Q H|}{\Lambda^{2}} \ln \left(\frac{\pi m^{2}}{|Q H|}\right)+\mathcal{O}(\epsilon)$

is valid only in the region near the critical value $\tau \rightarrow 1-0$. The closer $\tau$ to 1 , the smaller is $\epsilon$; Landau levels approach a continuun distribution, and a condensate occupies many levels. The physical reason for the changes found in the behaviour of the condensate is the strength of the four-fermion interaction which becomes essentially important here. The corresponding solution is

$M_{\text {dyn }}^{2}=\frac{|Q H|}{\pi} \exp \left[-\frac{\Lambda^{2}}{|Q H|}\left(\frac{1}{\tau}-1\right)\right]$.

Note that the near-critical regime found here, differs from the result of Ref. [5], being driven by a quadratic dependence on the cutoff, Eq. (24); this is tantamount of having a $(3+1)$ dimensional dynamics of fermion pairing.

Let us return now again to the three flavour case with $\kappa, g_{1}, g_{2} \neq 0$. In the simplest case with the octet flavour symmetry, where current quarks have equal masses $\hat{m}_{u}=\hat{m}_{d}=\hat{m}_{s}$, which we set again zero, the system (9) reduces to a cubic equation with respect to $h \equiv h_{u}=h_{d}=h_{s}$

$h^{3}+\frac{\kappa}{12 \lambda} h^{2}+\frac{4 G}{3 \lambda} h+\frac{4 m}{3 \lambda}=0$

with $\lambda=g_{1}+(2 / 3) g_{2}$. This cubic equation has one real root, if (see [20] for more details)

$\frac{G}{\lambda}>\left(\frac{\kappa}{24 \lambda}\right)^{2}$.

Assuming that the couplings fulfill condition (27), we find a single valued function $h(m)$ from Eq. (26).

Considering that most of the investigations have been using multi-quark Lagrangians without the stabilizing eight-quark interactions we make a short digression to discuss the case with $\lambda=0$ (see details in e.g. $[19,24]$ ). In this case Eq. (26) is quadratic with a regular and a singular solution as $\kappa \rightarrow 0$, i.e.,

$h^{(1,2)}=-\frac{8 G}{\kappa}\left(1 \mp \sqrt{1-\frac{\kappa m}{4 G^{2}}}\right)$.

In SPA this leads to an unstable effective potential. In the commonly used mean field approximation, which discards the singular solution, the effective potential is metastable and the region $4 G^{2}<\kappa m$ leads to complex values for $h$. This translates to

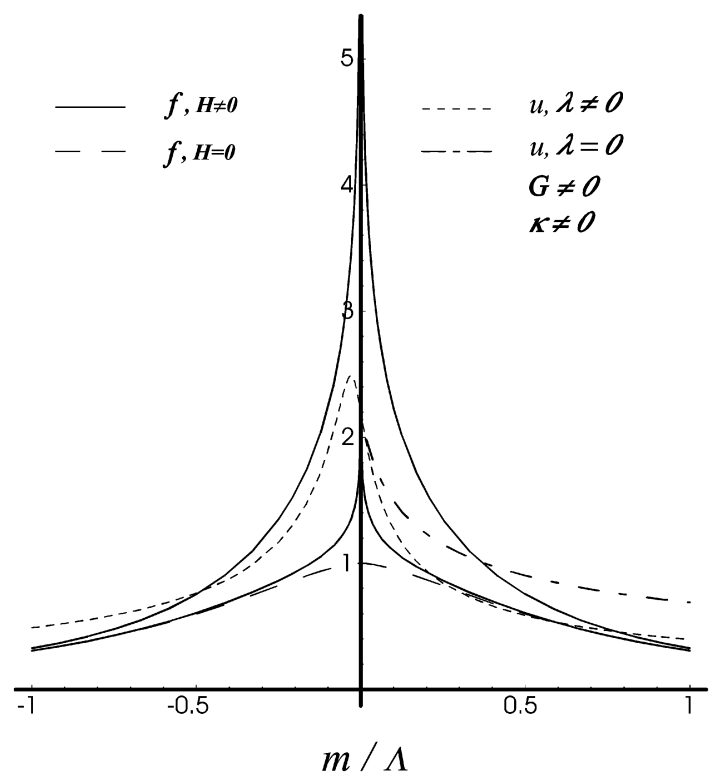

Fig. 2. The 1.h.s. and the r.h.s. of Eq. (29) as functions of $m / \Lambda$ with $f\left(m^{2} ; \Lambda,|Q H|\right)$ shown for $|Q H| \Lambda^{-2}=0 ; 0.1 ; 0.5$. The 1.h.s. with $\lambda \neq 0$ (short-dashed line) is shifted with respect to the ordinate axis due to the 't Hooft interaction, the range of values for the $\lambda=0$ curve (dash-dotted) is limited (see discussion in text). The value of $G \Lambda^{2}=3$ is the same as in Fig. 1. Here the sixand eight-quark couplings are taken to be $\kappa \Lambda^{5}=-10^{3}$ and $\lambda \Lambda^{8}=3.67 \times 10^{3}$ (or $\lambda=0$ ), respectively.

a restriction for the admissible values that the l.h.s. of Eq. (29) can assume, shown as dash-dotted line in Fig. 2, discussed below. Furthermore we find that qualitatively the symmetry breaking pattern is the same as in presence of the eight-quark interactions, but the occurrence of two minima requires higher values of $|\kappa|$, as compared to the case with $\lambda \neq 0(\sim 2.2 \kappa$ for the parameter set of Fig. 2). From now on we shall consider only the case which fulfills the stability requirement, provided by Eq. (27).

The nontrivial solutions for the mass of the fermion field in a constant magnetic field are determined by the equation

$-\frac{2 \pi^{2} h(m)}{\Lambda^{2} N_{c} m}=f\left(m^{2} ; \Lambda,|Q H|\right)$.

Comparing this result with Eq. (13), one sees that only the l.h.s. is changed. The six- and eight-quark interactions have modified it in such a way that now we get a function $h(m) / m$ instead of the former constant term involving only the coupling of fourquark interactions, $-1 / G$. The 1.h.s. of Eq. (29), abbreviated by $u$ in Fig. 2, has now a bell-shaped form (short-dashed line), to be compared with the horizontal line of Fig. 1. Note, that $h(m) / m=-1 / G+\mathcal{O}(m)$, i.e., the bell-shaped curve crosses the ordinate axis at the same point as the former straight line (for the same value of $G \Lambda^{2}$ ). The r.h.s. of Eq. (29) is again represented by the long-dashed curve $(H=0$ case $)$ and by the full lines for the finite $H$ cases: $|Q H| \Lambda^{-2}=0.1,0.5$. As mentioned before, these are not altered by the couplings $G, \kappa, \lambda$. The intersection points of the 1.h.s. with the r.h.s. curves yield the nontrivial solutions of the gap equation: one sees that either one or three solutions can be found for $m>0$. If Eq. (29) has no solutions at $H=0$ we say that the set of couplings $G, \kappa, \lambda$ 
are subcritical. It is said to be overcritical in the opposite case. Note that the overcritical set may contain $G<G_{\mathrm{cr}}$.

The trivial solution, $m=0$, corresponds to the point where the potential, $V(m)$, reaches its local maximum. Indeed, the second derivative

$\lim _{m \rightarrow 0} \frac{d^{2} V(m)}{d m^{2}}=\lim _{m \rightarrow 0} \frac{N_{c}|Q H|}{2 \pi^{2}} \ln \frac{|m| \Lambda}{2|Q H|}=-\infty$

is negative here. This is the general mathematical reason for the phenomenon known as magnetic catalysis of dynamical flavour symmetry breaking. The logarithmically divergent negative result ensures that this phase transition always takes place, if $H \neq 0$. This does not depend on the details related with the multi-quark dynamics, i.e., the result is true even for free fermions in a constant magnetic field.

What is really sensitive to the multi-quark dynamics is the local minima structure of the theory. Let us recall that in the theory with just four-fermion interactions the effective potential has only one minimum at $m>0$, and this property does not depend on the strength of the field $H$. We have demonstrated this in Fig. 1. In the theory with four-, six-, and eight-quark interactions one can find either one or two local minima at $m>0$. The result depends on the strength of the magnetic field $H$, and couplings $G, \kappa, \lambda$. We illustrate these two cases in Fig. 2. Namely, the upper full curve (r.h.s. of Eq. (29) for $|Q H| \Lambda^{-2}=0.5$ ) has only one intersection point with the bell-shaped curve $u$ (1.h.s. of Eq. (29) for $G \Lambda^{2}=3, \kappa \Lambda^{5}=-1000, \lambda \Lambda^{8}=3670$ ). This point corresponds to a single vacuum state of the theory. The other full curve (r.h.s. of Eq. (29) for $|Q H| \Lambda^{-2}=0.1$ ) has three intersections with the same curve $u$. These intersections, successively, correspond to a local minimum, a local maximum and a further local minimum of the potential.

It is interesting to note that the first minimum catalyzed by a constant magnetic field (that is, a slowly varying field) is then smoothed out with increasing $H$. It ceases to exist at some critical value of $|Q H| \Lambda^{-2}$, from which on only the large $M_{\mathrm{dyn}}$ solution survives. This is shown in Fig. 3, for the parameter set of Fig. 2. This process is accompanied by a sharp increase in depth of the effective potential at the second minimum, especially if we had at the beginning the opposite ordering, i.e., $V\left(M_{1}\right)<V\left(M_{2}\right)$. The reasons for such a synchronized behaviour are the following two facts. The first one is Eq. (30), which teaches us that the only way to wash out the first minimum is by lowering the barrier between this state and the second minimum. The other fact is the observation that the second minimum is unremovable, because the asymptotic behaviour of the functions in Eq. (29) is such that the 1.h.s. dominates over the r.h.s. at large $m / \Lambda$. This can also be understood from Fig. 2, where one sees that the r.h.s. of the gap equation with $H \neq 0$ approaches the $H=0$ curve from above.

To discuss the physical content of the phenomenon just described one should fix the characteristic scale $\Lambda$. We assume that this value is determined by the problem under consideration. Its choice can also be motivated by the number of Landau levels to be considered.

In the region $m^{2} / \Lambda^{2} \ll 1$ the four-fermion interaction dominates the behaviour of the system. Since their coupling strength

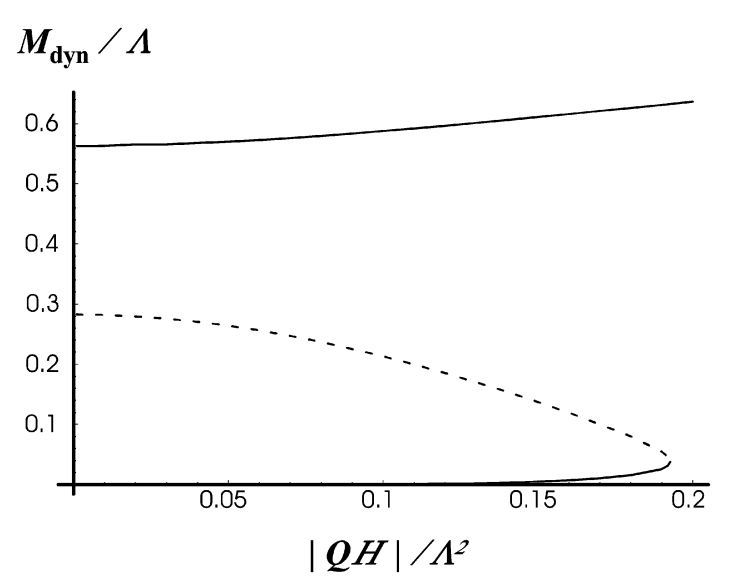

Fig. 3. The dimensionless dynamical mass $M_{\mathrm{dyn}} / \Lambda$ as a function of the dimensionless magnetic field $|Q H| / \Lambda^{2}$. The full lines are minima, the dashed line maxima. Up to $|Q H| / \Lambda^{2}=0.084$ the smaller $M_{\mathrm{dyn}} / \Lambda$ corresponds to the deeper minimum of the potential, from this value on the larger solution becomes the stable configuration.

is small, $G<G_{\mathrm{cr}}$, the massless fermions behave like almost free particles moving in a weak external magnetic field, with access to a large number of Landau levels, $\xi \gg 1$. This field catalyzes the process of fermion-antifermion pairing on the energy surface $E_{0}=0$ of the LLL. The first minimum localized at $m^{2} / \Lambda^{2} \ll 1$ is exactly formed by such a $(1+1)$-dimensional condensate. If six- and eight-fermion forces would not act on the system, this ground state would be stable: our formulae as well as the result of paper [5] show clearly that a slow increase of the strength $H$ does not wash out the condensate from the energy surface $E_{0}=0$ of the LLL. However, when the six- and eight-fermion interactions are present, a slowly increasing magnetic field destroys finally this ground state. The new condensate has a $(3+1)$-dimensional structure similar in every respect to the standard NJL case with broken chiral symmetry at $H=0$, i.e., when the condensate spreads over many single fermion states. This is because the increasing magnetic field enlarges the dynamical fermion mass, and scales of order $m / \Lambda \sim 1$ become relevant. At these scales the 't Hooft and eight-quark interactions push the system to a new regime, where the fermions are not anymore free-like particles: they interact strongly with each other and this interaction changes the fermionic spectrum and the structure of the ground state in an essential way, with all the above mentioned consequences.

Thus we have obtained not only a correct description of the well-known physics related with the LLL, but have found also a clear signature for the possibly important role played by 't Hooft and eight-quark interactions. Namely, in the presence of these interactions the magnetic field can change the condensation zone from the zero-energy surface of the LLL to a wide region, spread over many Landau levels and vice versa. One can expect that hard gamma emissions accompany this process.

What is the characteristic scale of the magnetic fields which can induce such a transition? We obtained the value $H=$ $7.3 \times 10^{13} \Lambda^{2} \mathrm{G} / \mathrm{MeV}^{2}$ which actually depends on the cutoff involved in the problem. For instance, in hadronic matter 
it is probably safe to assume that $\Lambda \simeq 800 \mathrm{MeV}$, leading to $H=4.7 \times 10^{19} \mathrm{G}$.

One can indicate several potentially interesting areas where this effect may find applications. One of them is connected with the recent studies of compact stellar objects in presence of strong magnetic fields, in particular the young neutron stars, magnetars [25]. The surface magnetic fields are observed to be $\geqslant 10^{15} \mathrm{G}$, but actually they can be even much higher in the core region. The other area is connected with the electroweak phase transition in the early Universe [26], where the strength of magnetic fields can reach $H \sim 10^{24} \mathrm{G}$.

\section{Acknowledgements}

This work has been supported in part by grants provided by Fundação para a Ciência e a Tecnologia, POCI/FP/63412/2005, $\mathrm{POCI} / \mathrm{FP} / 63930 / 2005$. This research is part of the EU integrated infrastructure initiative Hadron Physics project under contract No. RII3-CT-2004-506078.

\section{References}

[1] S.P. Klevansky, R.H. Lemmer, Phys. Rev. D 39 (1989) 3478.

[2] K.G. Klimenko, Theor. Math. Phys. 89 (1991) 211; K.G. Klimenko, Theor. Math. Phys. 90 (1992) 3;

A.S. Vshivtsev, K.G. Klimenko, B.V. Magnitsky, Theor. Math. Phys. 106 (1996) 390.

[3] I.V. Krive, S.A. Naftulin, Sov. J. Nucl. Phys. 54 (1991) 897; I.V. Krive, S.A. Naftulin, Phys. Rev. D 46 (1992) 2737.

[4] V.P. Gusynin, V.A. Miransky, I.A. Shovkovy, Phys. Rev. Lett. 73 (1994) 3499.

[5] V.P. Gusynin, V.A. Miransky, I.A. Shovkovy, Phys. Lett. B 349 (1995) 477;

V.P. Gusynin, V.A. Miransky, I.A. Shovkovy, Phys. Rev. D 52 (1995) 4718.

[6] V.P. Gusynin, V.A. Miransky, I.A. Shovkovy, Nucl. Phys. B 462 (1996) 249.

[7] J. Bardeen, L.N. Cooper, J.R. Schrieffer, Phys. Rev. 108 (1957) 1175.

[8] R. Jackiw, Phys. Rev. D 29 (1984) 2375; A. Barducci, R. Casalbuoni, L. Lusanna, Nuovo Cimento A 35 (1976) 377.

[9] R. Ragazzon, Phys. Lett. B 334 (1994) 427;

G. Dunne, T. Hall, Phys. Rev. D 53 (1996) 2220.

[10] R. Ragazzon, Phys. Rev. D 59 (1999) 065006.
[11] Y. Nambu, G. Jona-Lasinio, Phys. Rev. 122 (1961) 345; Y. Nambu, G. Jona-Lasinio, Phys. Rev. 124 (1961) 246; V.G. Vaks, A.I. Larkin, Zh. Eksp. Teor. Fiz. 40 (1961) 282.

[12] T. Eguchi, Phys. Rev. D 14 (1976) 2755; K. Kikkawa, Prog. Theor. Phys. 56 (1976) 947.

[13] M.K. Volkov, D. Ebert, Sov. J. Nucl. Phys. 36 (1982) 736; D. Ebert, M.K. Volkov, Z. Phys. C 16 (1983) 205.

[14] M.K. Volkov, Ann. Phys. 157 (1984) 282; A. Dhar, S. Wadia, Phys. Rev. Lett. 52 (1984) 959; A. Dhar, R. Shankar, S. Wadia, Phys. Rev. D 31 (1985) 3256; D. Ebert, H. Reinhardt, Nucl. Phys. B 271 (1986) 188; C. Schüren, E.R. Arriola, K. Goeke, Nucl. Phys. A 547 (1992) 612; J. Bijnens, C. Bruno, E. de Rafael, Nucl. Phys. B 390 (1993) 501, hepph/9206236;

V. Bernard, A.A. Osipov, U.-G. Meißner, Phys. Lett. B 324 (1994) 201, hep-ph/9312203;

V. Bernard, A.H. Blin, B. Hiller, Yu.P. Ivanov, A.A. Osipov, U.-G. Meißner, Ann. Phys. 249 (1996) 499, hep-ph/9506309.

[15] V. Bernard, R.L. Jaffe, U.-G. Meissner, Phys. Lett. B 198 (1987) 92; V. Bernard, R.L. Jaffe, U.-G. Meissner, Nucl. Phys. B 308 (1988) 753.

[16] H. Reinhardt, R. Alkofer, Phys. Lett. B 207 (1988) 482.

[17] S. Klimt, M. Lutz, U. Vogl, W. Weise, Nucl. Phys. A 516 (1990) 429; U. Vogl, M. Lutz, S. Klimt, W. Weise, Nucl. Phys. A 516 (1990) 469; U. Vogl, W. Weise, Prog. Part. Nucl. Phys. 27 (1991) 195.

[18] S.P. Klevansky, Rev. Mod. Phys. 64 (1992) 649; T. Hatsuda, T. Kunihiro, Phys. Rep. 247 (1994) 221, hep-ph/9401310.

[19] A.A. Osipov, B. Hiller, J. da Providência, Phys. Lett. B 634 (2006) 48, hep-ph/0508058.

[20] A.A. Osipov, B. Hiller, A.H. Blin, J. da Providência, Ann. Phys. (N.Y.) (2007), doi:10.1016/j.aop.2006.08.004, hep-ph/0607066.

[21] G. 't Hooft, Phys. Rev. D 14 (1976) 3432; G. 't Hooft, Phys. Rev. D 18 (1978) 2199, Erratum.

[22] J. Schwinger, Phys. Rev. 82 (1951) 664.

[23] H. Bateman, A. Erdelyi, Higher Transcendental Functions, McGraw-Hill, 1953.

[24] A.A. Osipov, B. Hiller, V. Bernard, A.H. Blin, Ann. Phys. 321 (2006) 2504, hep-ph/0507226;

A.A. Osipov, B. Hiller, V. Bernard, A.H. Blin, SIGMA 2 (2006) 026, hep$\mathrm{ph} / 0602165$.

[25] R.C. Duncan, C. Thompson, Astrophys. J. Lett. 392 (1992) L9; C. Thompson, R.C. Duncan, Astrophys. J. 408 (1993) 194;

C. Thompson, R.C. Duncan, Astrophys. J. 473 (1996) 322;

C. Kouveliotou, et al., Nature 391 (1999) 235;

K. Hurley, et al., Astrophys. J. 442 (1999) 111; P.M. Woods, et al., Astrophys. J. Lett. 519 (1999) L139.

[26] T. Vachaspati, Phys. Lett. B 265 (1991) 258; P. Olesen, Phys. Lett. B 281 (1992) 300. 